\title{
Character Formulas for Simple Modules of Hamiltonian Lie Superalgebras of Odd Type
}

\author{
Wende Liu, Jixia Yuan* and Shujuan Wang
}

\begin{abstract}
In this paper, character formulas are explicitly characterized for all simple restricted modules of Hamiltonian Lie superalgebras of odd type over an algebraically closed field of characteristic $p>3$. In the process we use the lengths and highest weights of simple quotients of restricted Kac modules of atypical weights with respect to a series of Borel subalgebras to determine the composition factors, composition series and the character formulas for the restricted Kac modules of atypical weights for the Lie superalgebras under consideration.
\end{abstract}

\section{Introduction}

Restricted Lie superalgebras and their restricted representations play a central role in the theory of modular Lie superalgebras, just as in the modular Lie algebra situation. A modular Lie superalgebra is referred to be restricted if its Lie algebra is restricted and the adjoint representation of its Lie algebra on the odd part is restricted. Let $L=L_{\overline{0}} \oplus L_{\overline{1}}$ be a restricted Lie superalgebra. The $p$-mapping $[p]$ of Lie algebra $L_{\overline{0}}$ is also called the $p$-mapping of the whole Lie superalgebra $L$. An $L$-module $M$ is called restricted provided that

$$
x^{p} \cdot m=x^{[p]} \cdot m \text { for all } x \in L_{\overline{0}}, m \in M .
$$

Over an algebraically closed field of characteristic $p>3$, there are four series of finitedimensional graded simple Lie superalgebras, called the generalized Witt, the special, the Hamiltonian and the contact Lie superalgebras, respectively, which are analogous to the corresponding four series of finite-dimensional graded simple modular Lie algebras of Cartan type [17]. Modular representations of these four series of Lie superalgebras have

Received May 4, 2018; Accepted November 19, 2018.

Communicated by Ching Hung Lam.

2010 Mathematics Subject Classification. 17B10, 17B50, $17 \mathrm{~B} 66$.

Key words and phrases. Hamiltonian Lie superalgebras of odd type, restricted Kac modules, atypical weights, character formulas.

Liu was supported by the National Natural Science Foundation of China (11471090).

Yuan was supported by the National Natural Science Foundation of China (11601135, 11771069), Natural

Science Foundation of Heilongjiang Province of China (QC2017002), Project funded by China Postdoctoral Science Foundation.

Wang was supported by the National Natural Science Foundation of China $(11701158,11501151)$.

*Corresponding author. 
been sufficiently studied by many authors (for example, see $10-15]$ ). Apart from these four series of graded simple Lie superalgebras, there are additionally four infinite series of finite-dimensional graded simple Lie superalgebras over an algebraically closed field of characteristic $p>3$, called the Hamiltonian Lie superalgebras of odd type, the special Hamiltonian Lie superalgebras of odd type, the contact Lie superalgebras of odd type and the special contact Lie superalgebras of odd type [1,7,8, which are analogous to the four series of infinite-dimensional simple Lie superalgebras of vector fields over $\mathbb{C}$ defined by odd differential forms [4]. Note that the latter four series of Lie superalgebras possess more complicated structures and have no analogues in Lie algebra case. In 2014, the authors obtained a sufficient and necessary condition for the restricted Kac modules to be simple for the restricted Hamiltonian Lie superalgebras of odd type over an algebraically closed field of characteristic $p>3[16$.

Let $\mathfrak{g}$ be a Hamiltonian Lie superalgebra of odd type over an algebraically closed field of characteristic $p>3$. In [16] root reflections are used to construct a series of Borel subalgebras of $\mathfrak{g}$ and to observe how the highest weights for simple quotients of restricted Kac modules change along with the Borel subalgebras of $\mathfrak{g}$ (see Lemma 3.1 below). Moreover, a group action on restricted Kac modules of $\mathfrak{g}$ is also introduced, which is consistent with the module action of Lie superalgebra $\mathfrak{g}$ itself and then the lengths of simple quotients are determined for the restricted Kac modules of $\mathfrak{g}$ with atypical weights (see Lemma 3.3). In this paper, we use the lengths and highest weights of simple quotients of restricted Kac modules of $\mathfrak{g}$ with atypical weights with respect to a series of Borel subalgebras to determine the composition factors, composition series and the character formulas for the restricted Kac modules of $\mathfrak{g}$ with atypical weights (see Theorem 4.4). Since a simple restricted module of $\mathfrak{g}$ is necessarily isomorphic to a simple quotient of a restricted Kac module of $\mathfrak{g}$, all simple restricted modules of $\mathfrak{g}$ are determined in a sense. We should mention that our methods are close to the ones used by Serganova for Cartan type Lie superalgebras over a field of characteristic zero $[9$ and by Shu and Zhang for Witt type Lie superalgebras over a field of prime characteristic [11, 12].

\section{Basics}

The ground field $\mathbb{F}$ is assumed to be algebraically closed and of characteristic $p>3$ and its prime subfield is denoted by $\mathbb{F}_{p}$. All algebras, modules are assumed to be finitedimensional, unless specified otherwise. Denote by $\mathbb{Z}_{2}=\{\overline{0}, \overline{1}\}$ the additive group of order two. For a vector superspace $V=V_{\overline{0}} \oplus V_{\overline{1}}$, write $|x|$ for the parity of a homogeneous element $x$ in $V$. The symbol $|x|$ implies that $x$ is already assumed to be a homogeneous element. We also adopt the following notation: For a proposition $P$, put $\delta_{P}=1$ if $P$ is true and $\delta_{P}=0$ otherwise. 
By definition, the restricted enveloping algebra $\mathbf{u}(L)$ of a restricted Lie superalgebra $(L,[p])$ is the quotient algebra of the universal enveloping algebra $\mathbf{U}(L)$ modulo the twosided ideal generated by all elements $x^{p}-x^{[p]}$ with $x \in L_{\overline{0}}$.

\subsection{Divided power superalgebras}

Fix a pair of positive integers $m, n$ and write $\underline{r}=\left(r_{1}, \ldots, r_{m} \mid r_{m+1}, \ldots, r_{m+n}\right)$ for an $(m, n)$-tuple of non-negative integers. For an $m$-tuple of positive integers, $\underline{N}=$ $\left(N_{1}, \ldots, N_{m}\right)$, let $\mathbf{I}(m, \underline{N} \mid n)$ be the set of all $(m, n)$-tuples $\underline{r}$ such that $r_{i}<p^{N_{i}}$ for $1 \leq i \leq m$ and $r_{i}=0$ or 1 for $m<i \leq m+n$. Following [5, 6], write $\mathcal{O}(m, \underline{N} \mid n)$ for the divided power superalgebras, which is a supercommutative associative superalgebra having a basis $\left\{x^{(\underline{r})} \mid \underline{r} \in \mathbf{I}(m, \underline{N} \mid n)\right\}$ with parity $\left|x^{(\underline{r})}\right|=\left(\sum_{i>m} r_{i}\right) \overline{1}$ and multiplication:

$$
x^{(\underline{r})} x^{(\underline{s})}=\prod_{i=m+1}^{m+n} \min \left(1,2-r_{i}-s_{i}\right)(-1)^{\sum_{m<i<j \leq m+n} r_{j} s_{i}}\left(\begin{array}{c}
\underline{r}+\underline{s} \\
\underline{r}
\end{array}\right) x^{(\underline{r}+\underline{s})} .
$$

Note that $\mathcal{O}(m, \underline{N} \mid n)$ is a generalization of the divided power algebra $\mathcal{O}(m, \underline{N})$ and is isomorphic to the tensor product the divided power algebra with the trivial $\mathbb{Z}_{2}$-grading and the exterior algebra of rank $n$ with the natural $\mathbb{Z}_{2}$-grading:

$$
\mathcal{O}(m, \underline{N} \mid n) \simeq \mathcal{O}(m, \underline{N}) \otimes \Lambda(n)
$$

\subsection{Hamiltonian Lie superalgebras of odd type}

Let $\epsilon_{i}$ be the $(m+n)$-tuple with 1 in the $i$-th place and 0 elsewhere. For simplicity, write $x_{i}$ for $x^{\left(\epsilon_{i}\right)}$. Define the distinguished partial derivative $\partial_{i}$ with parity $\left|\partial_{i}\right|=\left|x_{i}\right|$ by letting

$$
\partial_{i}\left(x_{j}\right)=\delta_{i j} \quad \text { for } 1 \leq i, j \leq m+n
$$

From now on, suppose $m=n$. As in 5, 6], write

$$
\operatorname{De}_{f}=\sum_{i=1}^{2 n}(-1)^{\left|\partial_{i} \| f\right|} \partial_{i}(f) \partial_{i^{\prime}}
$$

where

$$
i^{\prime}= \begin{cases}i+n & \text { if } 1 \leq i \leq n \\ i-n & \text { if } n<i \leq 2 n\end{cases}
$$

Note that

$$
\left|\operatorname{De}_{f}\right|=|f|+\overline{1}
$$

and

$$
\left[\operatorname{De}_{f}, \operatorname{De}_{g}\right]=\operatorname{De}_{\{f, g\}_{B}} \quad \text { for all } f, g \in \mathcal{O}(n, \underline{N} \mid n)
$$


where $\{\cdot, \cdot\}_{B}$ is the Buttion bracket given by

$$
\{f, g\}_{B}=\operatorname{De}_{f}(g)=\sum_{i=1}^{2 n}(-1)^{\left|\partial_{i}\right||f|} \partial_{i}(f) \partial_{i^{\prime}}(g)
$$

Then

$$
\mathfrak{l} \mathfrak{e}(n, \underline{N} \mid n)=\left\{\operatorname{De}_{f} \mid f \in \mathcal{O}(n, \underline{N} \mid n)\right\}
$$

is a finite-dimensional simple Lie superalgebra, called the Hamiltonian Lie superalgebra of odd type. This Lie superalgebra was also called the odd Hamiltonian superalgebra and denoted by $\mathrm{HO}(n, n ; \underline{N})$ in $[8]$. In the present paper, we adopt the notation in [5, 6]. Note that it is analogous to the infinite-dimensional Lie superalgebra $\mathrm{HO}(n, n)$ of vector fields over $\mathbb{C}($ see $[4])$.

\subsection{Extension}

We extend $\mathfrak{l}(n, \underline{1} \mid n)$ to

$$
\overline{\mathfrak{l}}(n, \underline{1} \mid n)=\mathfrak{l} \mathfrak{e}(n, \underline{1} \mid n)+\mathbb{F} \sum_{i=1}^{2 n} x_{i} \partial_{i} .
$$

By letting $\operatorname{deg} x_{i}=1=-\operatorname{deg} \partial_{i}, \overline{\mathfrak{l}}(n, \underline{1} \mid n)$ becomes a $\mathbb{Z}$-graded Lie superalgebra

$$
\overline{\mathfrak{l}}(n, \underline{1} \mid n)=\bigoplus_{i \geq-1} \overline{\mathfrak{l} e}(n, \underline{1} \mid n)_{[i]}
$$

and the corresponding descending filtration is denoted by $\left(\overline{\mathfrak{l}}(n, \underline{1} \mid n)_{i}\right)_{i \geq-1}$. By abuse language, we also call $\overline{\mathfrak{l}}(n, \underline{1} \mid n)$ a Hamiltonian Lie superalgebra of odd type.

Note that $\mathfrak{l}(n, \underline{1} \mid n)$ is a $\mathbb{Z}$-graded subalgebra of $\overline{\mathfrak{l}}(n, \underline{1} \mid n)$ and $\sum_{i=1}^{2 n} x_{i} \partial_{i}$ is precisely the degree derivation of $\mathfrak{k}(n, \underline{1} \mid n)$. In this paper we aim to determine the character formulas for simple restricted modules of $\overline{\mathfrak{l}}(n, \underline{1} \mid n)$.

Convention. In the subsequent sections we will write $\mathfrak{g}$ for $\overline{\mathfrak{l}}(n, \underline{1} \mid n)$.

\subsection{Triangular decompositions}

Let $\overline{\mathfrak{h}}=\mathfrak{h} \oplus \mathbb{F} \sum_{i=1}^{2 n} x_{i} \partial_{i}$, where

$$
\mathfrak{h}=\operatorname{span}_{\mathbb{F}}\left\{\operatorname{De}_{x_{i} x_{i^{\prime}}} \mid 1 \leq i \leq n\right\} .
$$

Then $\overline{\mathfrak{h}}$ is a Cartan subalgebra of $\mathfrak{g}_{[0]}$ and $\mathfrak{g}=\bigoplus_{\alpha \in \overline{\mathfrak{h}}^{*}} \mathfrak{g}_{\alpha}$, where

$$
\mathfrak{g}_{\alpha}=\{x \in \mathfrak{g} \mid[h, x]=\alpha(h) x \text { for } h \in \overline{\mathfrak{h}}\} .
$$


Write the dual basis of $\overline{\mathfrak{h}}$ as follows:

$$
\varepsilon_{i}=\left(\operatorname{De}_{x_{i} x_{i^{\prime}}}\right)^{*}, \quad \delta=\left(\sum_{j=1}^{2 n} x_{j} \partial_{j}\right)^{*} \quad \text { for all } 1 \leq i \leq n .
$$

Clearly,

$$
\operatorname{De}_{x_{i}} \in \begin{cases}\mathfrak{g}_{-\varepsilon_{i}-\delta} & \text { if } 1 \leq i \leq n, \\ \mathfrak{g}_{\varepsilon_{i^{\prime}}-\delta} & \text { if } n<i \leq 2 n\end{cases}
$$

Note that $\mathfrak{g}_{[0]}$ has a standard triangular decomposition $\mathfrak{g}_{[0]}=\mathfrak{n}_{[0]}^{-} \oplus \overline{\mathfrak{h}} \oplus \mathfrak{n}_{[0]}^{+}$, where

$$
\begin{gathered}
\mathfrak{n}_{[0]}^{-}=\operatorname{span}_{\mathbb{F}}\left\{\operatorname{De}_{x_{i} x_{n+j}} \mid n \geq i>j \geq 1\right\}+\operatorname{span}_{\mathbb{F}}\left\{\operatorname{De}_{x_{k} x_{l}} \mid n<k, l \leq 2 n\right\}, \\
\mathfrak{n}_{[0]}^{+}=\operatorname{span}_{\mathbb{F}}\left\{\operatorname{De}_{x_{i} x_{n+j}} \mid 1 \leq i<j \leq n\right\}+\operatorname{span}_{\mathbb{F}}\left\{\operatorname{De}_{x_{k} x_{l}} \mid 1 \leq k, l \leq n\right\} .
\end{gathered}
$$

Then $\mathfrak{g}$ has a standard triangular decomposition $\mathfrak{g}=\mathfrak{n}_{0}^{-} \oplus \overline{\mathfrak{h}} \oplus \mathfrak{n}_{0}^{+}$, where

$$
\mathfrak{n}_{0}^{-}=\mathfrak{n}_{[0]}^{-} \oplus \mathfrak{g}_{[-1]}, \quad \mathfrak{n}_{0}^{+}=\mathfrak{n}_{[0]}^{+} \oplus \bigoplus_{i>0} \mathfrak{g}_{[i]} .
$$

As in 16, we define a sequence of root reflections in the order:

$$
\gamma_{-\varepsilon_{1}-\delta}, \ldots, \gamma_{-\varepsilon_{n}-\delta}, \gamma_{\varepsilon_{n}-\delta}, \ldots, \gamma_{\varepsilon_{1}-\delta}
$$

and then obtain a series of new triangular decompositions:

$$
\mathfrak{g}=\mathfrak{n}_{i}^{-} \oplus \overline{\mathfrak{h}} \oplus \mathfrak{n}_{i}^{+} \quad \text { for all } 1 \leq i \leq 2 n
$$

For $0 \leq i \leq 2 n$, put $\mathfrak{b}_{i}=\mathfrak{n}_{i}^{+} \oplus \overline{\mathfrak{h}}$. Then $\mathfrak{b}_{i}$ are Borel subalgebras containing $\mathfrak{b}_{[0]}$, where $\mathfrak{b}_{[0]}=\overline{\mathfrak{h}} \oplus \mathfrak{n}_{[0]}^{+}$is the canonical Borel subalgebra of $\mathfrak{g}_{[0]}$.

\subsection{Restricted Kac modules}

Suppose $\mathfrak{g}\left(\right.$ resp. $\left.\mathfrak{g}_{[0]}\right)$ has a triangular decomposition

$$
\mathfrak{g}=N^{-} \oplus \overline{\mathfrak{h}} \oplus N^{+} \quad\left(\text { resp. } \mathfrak{g}_{[0]}=N_{[0]}^{-} \oplus \overline{\mathfrak{h}} \oplus N_{[0]}^{+}\right) .
$$

Let $V=V_{\overline{0}} \oplus V_{\overline{1}}$ be a $\mathfrak{g}$-module (resp. $\mathfrak{g}_{[0]}$-module). If for $\lambda \in \overline{\mathfrak{h}}^{*}$ there is a nonzero vector $v \in V_{\overline{0}} \cup V_{\overline{1}}$ such that

$$
\begin{gathered}
h \cdot v=\lambda(h) v \quad \text { for all } h \in \overline{\mathfrak{h}} \\
x \cdot v=0 \quad \text { for all } x \in N^{+} \quad\left(\text { resp. } x \in N_{[0]}^{+}\right),
\end{gathered}
$$

then $v$ is called a highest weight vector in $V$ of highest weight $\lambda$ with respect to Borel subalgebra $B=\overline{\mathfrak{h}} \oplus N^{+}$(resp. $B_{[0]}=\overline{\mathfrak{h}} \oplus N_{[0]}^{+}$). If $V$ is a restricted $\mathfrak{g}$-module (resp. $\mathfrak{g}_{[0]^{-}}$ module) with weight $\lambda$, then $\lambda \in \mathbb{F}_{p}^{n+1}$, where $\mathbb{F}_{p}^{n+1}=\operatorname{span}_{\mathbb{F}_{p}}\left\{\varepsilon_{1}, \ldots, \varepsilon_{n}, \delta\right\}$. A weight 
$\lambda \in \mathbb{F}_{p}^{n+1}$ is called atypical if $\lambda \in \Omega$ and typical otherwise, where $\Omega$ consists of the following weights with $a, b \in \mathbb{F}_{p}, 1 \leq i \leq n$ :

$$
\begin{aligned}
\varepsilon_{i, a, b} & =\sum_{j=1}^{i-1} \varepsilon_{j}+a \varepsilon_{i}+(b+a+i-1) \delta, \\
\varepsilon_{i, b} & =\sum_{j=1}^{n} \varepsilon_{j}+\sum_{l=i}^{n} \varepsilon_{l}+(b+i-1) \delta .
\end{aligned}
$$

In the sequel, for $\lambda \in \overline{\mathfrak{h}}^{*}$, we write $\mathbb{F} v_{\lambda}$ for the 1-dimensional module of $\overline{\mathfrak{h}}$ with module action

$$
h \cdot v_{\lambda}=\lambda(h) v_{\lambda} \quad \text { for all } h \in \overline{\mathfrak{h}} .
$$

Write $L^{0}(\lambda)$ for the simple head of the restricted Verma module $\mathbf{u}\left(\mathfrak{g}_{[0]}\right) \bigotimes_{\mathbf{u}\left(\mathfrak{b}_{[0]}\right)} \mathbb{F} v_{\lambda}$. Note that every simple $\mathbf{u}\left(\mathfrak{g}_{[0]}\right)$-module is isomorphic to some $L^{0}(\lambda)$ with some highest weight $\lambda$ $\left(\right.$ see 3 ). Let $\mathfrak{b}$ be a Borel subalgebra of $\mathfrak{g}$ containing $\mathfrak{b}_{[0]}$. Then

$$
I^{\mathfrak{b}}(\lambda)=\mathbf{u}(\mathfrak{g}) \bigotimes_{\mathbf{u}\left(\mathfrak{b}+\mathfrak{g}_{[0]}\right)} L^{0}(\lambda)
$$

is called a restricted Kac module of $\mathfrak{g}$ with respect to $\mathfrak{b}$. Obviously $I^{\mathfrak{b}}(\lambda)$ is a $\mathbb{Z}$-graded $\mathfrak{g}$-module and $I^{\mathfrak{b}}(\lambda)$ has a unique simple quotient module, which will be denoted by $L^{\mathfrak{b}}(\lambda)$.

\section{6. $(\mathbf{u}(\mathfrak{g}), \mathfrak{T})$-modules}

Recall that the conformal symplectic supergroup $\operatorname{CSP}(n, \mathbb{F})$ is a direct product of the symplectic group $\operatorname{SP}(n, \mathbb{F})$ and the one-dimensional multiplicative supergroup $\mathbb{F}^{*}$. Let $\mathfrak{T}$ be the canonical maximal torus of the $\operatorname{CSP}(n, \mathbb{F})$ and $\chi(\mathfrak{T})$ be the character group of $\mathfrak{T}$. A rational $\mathfrak{T}$-module $V$ is by definition that $V=\bigoplus_{\lambda \in \chi(\mathfrak{T})} V_{\lambda}$, where

$$
V_{\lambda}=\{v \in V \mid \mathbf{t} \cdot v=\lambda(\mathbf{t}) v, \mathbf{t} \in \mathfrak{T}\} .
$$

Note that $\mathbf{u}(\mathfrak{g})$ and $\mathbf{u}\left(\mathfrak{g}_{[0]}\right)$ are rational $\mathfrak{T}$-modules $[16]$.

According to [11, a finite-dimensional superspace $V=V_{\overline{0}} \oplus V_{\overline{1}}$ is called a $(\mathbf{u}(\mathfrak{g}), \mathfrak{T})$ module if $V$ is both a $\mathbf{u}(\mathfrak{g})$-module and a rational $\mathfrak{T}$-module for which each $V_{\alpha}$ with $\alpha \in \mathbb{Z}_{2}$ is a $\mathfrak{T}$-module and the following statements hold:

(1) The actions of $\mathfrak{h}$ coming from $\mathfrak{g}$ and from $\mathfrak{T}$ coincide.

(2) $\mathbf{t} \cdot(a \cdot v)=(\mathbf{t} \cdot a) \cdot(\mathbf{t} \cdot v)$ for all $\mathbf{t} \in \mathfrak{T}, a \in \mathbf{u}(\mathfrak{g}), v \in V$.

Note that $I^{\mathfrak{b}_{i}}(\lambda)$ and $L^{\mathfrak{b}_{i}}(\lambda)$ are $(\mathbf{u}(\mathfrak{g}), \mathfrak{T})$-modules, where $0 \leq i \leq 2 n$ and $\lambda \in \mathbb{F}_{p}^{n+1}$ (see [16]). 


\section{Reduction lemmas}

For $1 \leq i \leq n$, put

$$
\mathfrak{h}_{i}:=\operatorname{span}_{\mathbb{F}}\left\{\operatorname{De}_{x_{j} x_{j^{\prime}}} \mid 1 \leq j \leq n, j \neq i\right\} .
$$

Clearly, for $\lambda \in \mathbb{F}_{p}^{n+1}$ and a fixed $i, \lambda\left(\mathfrak{h}_{i}\right)=0$ if and only if $\lambda$ is of one of the following forms $b \varepsilon_{i}+a \delta$, where $a, b \in \mathbb{F}_{p}$. From [16, Proposition 3.1], we have the following lemma. In the sequel, we identify two weight vectors of a weight if they are proportional.

Lemma 3.1. Let $\lambda \in \mathbb{F}_{p}^{n+1}, 1 \leq i \leq n$ and $v_{0}, \ldots, v_{2 n}$ be highest weight vectors of $L^{\mathfrak{b}_{0}}(\lambda)$ with respect to $\mathfrak{b}_{0}, \ldots, \mathfrak{b}_{2 n}$, respectively.

- If $\lambda\left(\mathfrak{h}_{i}\right) \neq 0$, then

$$
v_{i}=\mathrm{De}_{x_{i}} \cdot v_{i-1} \quad \text { and } \quad v_{(n-i+1)^{\prime}}=\mathrm{De}_{x_{i^{\prime}}}^{p-1} \cdot v_{(n-i)^{\prime}}
$$

In particular,

$$
L^{\mathfrak{b}_{i-1}}(\lambda) \cong L^{\mathfrak{b}_{i}}\left(\lambda-\varepsilon_{i}-\delta\right), \quad L^{\mathfrak{b}_{(n-i)^{\prime}}}(\lambda) \cong L^{\mathfrak{b}_{(n-i+1)^{\prime}}}\left(\lambda-\varepsilon_{i}+\delta\right) .
$$

- If $\lambda\left(\mathfrak{h}_{i}\right)=0$, then $v_{i}=v_{i-1}$ and

$$
v_{(n-i+1)^{\prime}}= \begin{cases}v_{(n-i)^{\prime}} & \lambda=a \delta, a \in \mathbb{F}_{p}, \\ \operatorname{De}_{x_{i^{\prime}}}^{p-2} \cdot v_{(n-i)^{\prime}} & \lambda=\varepsilon_{i}+a \delta, a \in \mathbb{F}_{p}, \\ \operatorname{De}_{x_{i^{\prime}}}^{p-1} \cdot v_{(n-i)^{\prime}} & \lambda=b \varepsilon_{i}+a \delta, a, b \in \mathbb{F}_{p}, b \neq 0,1 .\end{cases}
$$

In particular,

$$
L^{\mathfrak{b}_{i-1}}(\lambda) \cong L^{\mathfrak{b}_{i}}(\lambda)
$$

and

$$
L^{\mathfrak{b}_{(n-i)^{\prime}}}(\lambda) \cong \begin{cases}L^{\mathfrak{b}_{(n-i+1)^{\prime}}}(\lambda) & \lambda=a \delta, a \in \mathbb{F}_{p}, \\ L^{\mathfrak{b}_{(n-i+1)^{\prime}}\left(\lambda-2\left(\varepsilon_{i}-\delta\right)\right)} & \lambda=\varepsilon_{i}+a \delta, a \in \mathbb{F}_{p}, \\ L^{\mathfrak{b}_{(n-i+1)^{\prime}}}\left(\lambda-\varepsilon_{i}+\delta\right) & \lambda=b \varepsilon_{i}+a \delta, a, b \in \mathbb{F}_{p}, b \neq 0,1 .\end{cases}
$$

The following lemma was obtained in [16, Theorem 1]. However, for the reader's convenience, we give a proof with more clear explanations.

Lemma 3.2. Let $\lambda \in \mathbb{F}_{p}^{n+1}$. Then $\mathfrak{g}$-module $I^{\mathfrak{b}_{0}}(\lambda)$ is simple if and only if $\lambda$ is typical.

Proof. Let $v_{0}$ and $v_{2 n}$ be highest weight vectors of $L^{\mathfrak{b}_{0}}(\lambda)$ with respect to $\mathfrak{b}_{0}$ and $\mathfrak{b}_{2 n}$, respectively. Note that highest weight vectors of $L^{\mathfrak{b}_{0}}(\lambda)$ with respect to $\mathfrak{b}_{0}$ are proportional and the nonzero homomorphic image of a highest weight vector of $I^{\mathfrak{b}_{0}}(\lambda)$ with 
respect to $\mathfrak{b}_{0}$ is also a highest weight vector. Then $v_{0}$ can be viewed as a canonical homomorphic image of $v_{\lambda}$. Note that any nonzero submodule of $I^{\mathfrak{b}_{0}}(\lambda)$ contains $\operatorname{De}_{x_{1^{\prime}}}^{p-1} \cdots \operatorname{De}_{x_{n^{\prime}}}^{p-1} \operatorname{De}_{x_{n}} \cdots \operatorname{De}_{x_{1}} v_{\lambda}$. Then $I^{\mathfrak{b}_{0}}(\lambda)$ is simple if and only if

$$
v_{2 n}=\operatorname{De}_{x_{1^{\prime}}}^{p-1} \cdots \operatorname{De}_{x_{n^{\prime}}}^{p-1} \operatorname{De}_{x_{n}} \cdots \operatorname{De}_{x_{1}} \cdot v_{0}
$$

Then it is sufficient to show that $\lambda$ is atypical if and only if

$$
v_{2 n} \neq \operatorname{De}_{x_{1^{\prime}}}^{p-1} \cdots \operatorname{De}_{x_{n^{\prime}}}^{p-1} \operatorname{De}_{x_{n}} \cdots \operatorname{De}_{x_{1}} \cdot v_{0}
$$

By Lemma 3.1, one may express $v_{2 n}$ by $v_{0}$ and elements of $\mathbf{u}\left(\mathfrak{g}_{[-1]}\right)$, that is, there exists $x \in \mathbf{u}\left(\mathfrak{g}_{[-1]}\right)$ such that $v_{2 n}=x \cdot v_{0}$. Consequently, $\lambda$ is atypical if and only if

$$
v_{2 n} \neq \mathrm{De}_{x_{1^{\prime}}}^{p-1} \cdots \mathrm{De}_{x_{n^{\prime}}}^{p-1} \mathrm{De}_{x_{n}} \cdots \mathrm{De}_{x_{1}} \cdot v_{0} .
$$

Set

$$
\mathfrak{J}=\left\{\operatorname{diag}(1, \ldots, 1, t) \mid t \in \mathbb{F}^{*}\right\} .
$$

Since we have the following group isomorphism

$$
\mathfrak{T} \cong\left\{\operatorname{diag}\left(t_{1}, \ldots, t_{n}, t_{1}^{-1}, \ldots, t_{n}^{-1}, t\right) \mid t, t_{i} \in \mathbb{F}^{*}\right\}
$$

$\mathfrak{J}$ can be viewed as a subgroup of $\mathfrak{T}$.

Note that any rational $\mathfrak{T}$-module $V$ has a $\mathbb{Z}$-grading decomposition $V=\bigoplus_{s \in \mathbb{Z}} V_{s}$, where

$$
V_{s}=\left\{v \in V \mid \mathbf{t}(v)=t^{s} v, \mathbf{t}=\operatorname{diag}(1, \ldots, 1, t) \in \mathfrak{J}\right\} .
$$

Let $L=L_{\overline{0}} \oplus L_{\overline{1}}$ be an abelian Lie superalgebra with the trivial $p$-mapping, $\operatorname{dim} L_{\overline{0}}=m$ and $\operatorname{dim} L_{\overline{1}}=n$. Then we have the following superalgebra isomorphism

$$
\mathbf{u}(L) \cong\left(\mathbb{F}\left[x_{1}, \ldots, x_{m}\right] /\left\langle x_{1}^{p}, \ldots, x_{m}^{p}\right\rangle\right) \otimes \Lambda(n)
$$

where $\left\langle x_{1}^{p}, \ldots, x_{m}^{p}\right\rangle$ is the two-sided ideal of the polynomial algebra $\mathbb{F}\left[x_{1}, \ldots, x_{m}\right]$ generated by $x_{1}^{p}, \ldots, x_{m}^{p}$. So $\mathbf{u}(L)$ has a natural $\mathbb{Z}$-grading structure induced by the standard $\mathbb{Z}$ grading structures of $\mathbb{F}\left[x_{1}, \ldots, x_{m}\right]$ and $\Lambda(n)$. Let $\lambda=\sum_{i=1}^{n} a_{i} \varepsilon_{i}+a \delta$, where $a_{i}, a \in \mathbb{F}_{p}$. Then

$$
I^{\mathfrak{b}_{0}}(\lambda)=\bigoplus_{i=a-p n}^{a} I^{\mathfrak{b}_{0}}(\lambda)_{i}, \quad L^{\mathfrak{b}_{0}}(\lambda)=\bigoplus_{i=a-p n}^{a} L^{\mathfrak{b}_{0}}(\lambda)_{i}
$$

where

$$
I^{\mathfrak{b}_{0}}(\lambda)_{i}=\mathbf{u}\left(\mathfrak{g}_{[-1]}\right)_{a-i} \mathbf{u}\left(\mathfrak{g}_{[0]}\right) v_{\lambda}, \quad L^{\mathfrak{b}_{0}}(\lambda)_{i}=\mathbf{u}\left(\mathfrak{g}_{[-1]}\right)_{a-i} \mathbf{u}\left(\mathfrak{g}_{[0]}\right) \cdot v_{\lambda}
$$

Put

$$
\operatorname{supp}_{\mathfrak{J}}(V)=\left\{s \in \mathbb{Z} \mid V_{s} \neq 0\right\}
$$


For any $(\mathbf{u}(\mathfrak{g}), \mathfrak{T})$-module $V$, we define the length of $V$ to be the number $\left|\operatorname{supp}_{\mathfrak{J}}(V)\right|$ minus 1 and denote it by $\operatorname{len}(V)$. Then $\operatorname{len}\left(I^{\mathfrak{b}_{0}}(\lambda)\right)=p n$ and $\operatorname{len}\left(L^{\mathfrak{b}_{0}}(\lambda)\right) \leq p n$. If $\lambda$ is a typical weight, then $I^{\mathfrak{b}_{0}}(\lambda) \cong L^{\mathfrak{b}_{0}}(\lambda)$ and so $\operatorname{len}\left(L^{\mathfrak{b}_{0}}(\lambda)\right)=p n$.

The following lemma is already contained in the proof of [16, Theorem 1]. However, for the reader's convenience, we also give a proof.

Lemma 3.3. Let $\lambda$ be an atypical weight. Then

$$
\operatorname{len}\left(L^{\mathfrak{b}_{0}}(\lambda)\right)= \begin{cases}0 & \text { if } \lambda=a \delta, a \in \mathbb{F}_{p}, \\ p n-2 & \text { if } \lambda=\varepsilon_{n, 1, a}, a \in \mathbb{F}_{p}, \\ p n-1 & \text { otherwise. }\end{cases}
$$

Proof. Let $v_{0}$ and $v_{2 n}$ be highest weight vectors of $L^{\mathfrak{b}_{0}}(\lambda)$ with respect to $\mathfrak{b}_{0}$ and $\mathfrak{b}_{2 n}$, respectively. Write $L^{\mathfrak{b}_{0}}(\lambda)=\bigoplus_{i=l}^{h} L^{\mathfrak{b}_{0}}(\lambda)_{i}$ and $\lambda=\sum_{i=1}^{n} a_{i} \varepsilon_{i}+a \delta$ with $a_{i}, a \in \mathbb{F}_{p}$. As in the proof of Lemma 3.2 , we can view $v_{0}$ as a canonical homomorphic image of $v_{\lambda}$. Then we have $v_{0} \in L^{\mathfrak{b}_{0}}(\lambda)_{a}$ and therefore $h=a$. By Lemma 3.1, we have

$$
v_{2 n} \in \begin{cases}L^{\mathfrak{b}_{0}}(\lambda)_{a} & \text { if } \lambda=a \delta, a \in \mathbb{F}_{p}, \\ L^{\mathfrak{b}_{0}}(\lambda)_{a-p n+2} & \text { if } \lambda=\varepsilon_{n, 1, a}, a \in \mathbb{F}_{p} \\ L^{\mathfrak{b}_{0}}(\lambda)_{a-p n+1} & \text { otherwise. }\end{cases}
$$

Since $\mathfrak{b}_{2 n}=\mathfrak{b}_{[0]} \oplus \mathfrak{g}_{[-1]}$, we have $\mathbf{u}\left(\mathfrak{g}_{[-1]}\right) \mathfrak{g}_{[-1]} \cdot v_{2 n}=0$. It follows that

$$
l= \begin{cases}a & \text { if } \lambda=a \delta, a \in \mathbb{F}_{p}, \\ a-p n+2 & \text { if } \lambda=\varepsilon_{n, 1, a}, a \in \mathbb{F}_{p}, \\ a-p n+1 & \text { otherwise. }\end{cases}
$$

The proof is complete.

Remark 3.4. Let $\lambda$ be an atypical weight. Then any simple subquotient of $I^{\mathfrak{b}_{0}}(\lambda)$ must be $L^{\mathfrak{b}_{0}}(\mu)$ for some atypical weight $\mu$. To see this, it is sufficient to show that $L^{\mathfrak{b}_{0}}(\nu)$ is not a simple subquotient of $I^{\mathfrak{b}_{0}}(\lambda)$ for any typical weight $\nu$. By Lemma 3.2 , one sees that $I^{\mathfrak{b}_{0}}(\nu)=L^{\mathfrak{b}_{0}}(\nu)$. Then $\operatorname{len}\left(L^{\mathfrak{b}_{0}}(\nu)\right)=\operatorname{len}\left(I^{\mathfrak{b}_{0}}(\nu)\right)$. Since $\operatorname{len}\left(I^{\mathfrak{b}_{0}}(\nu)\right)=\operatorname{len}\left(I^{\mathfrak{b}_{0}}(\lambda)\right)=p n$, $L^{\mathfrak{b}_{0}}(\nu)$ is not a simple subquotient $I^{\mathfrak{b}_{0}}(\lambda)$.

For any fixed $i$ with $0 \leq i \leq 2 n,\left\{L^{\mathfrak{b}_{i}}(\lambda) \mid \lambda \in \mathbb{F}_{p}^{n+1}\right\}$ constitute the set of iso-classes of simple restricted $\mathfrak{g}$-modules. Hence for any $\lambda \in \mathbb{F}_{p}^{n+1}$, there is unique $\lambda^{\prime} \in \mathbb{F}_{p}^{n+1}$ such that $L^{\mathfrak{b}_{0}}(\lambda) \cong L^{\mathfrak{b}_{2 n}}\left(\lambda^{\prime}\right)$. Write $\operatorname{mult}(\lambda, \mu)$ for the multiplicity of $L^{\mathfrak{b}_{0}}(\mu)$ in $I^{\mathfrak{b}_{0}}(\lambda)$, where $\lambda, \mu \in \mathbb{F}_{p}^{n+1}$. The ingredient $\operatorname{mult}(\lambda, \mu)$ is crucial for computing the character formulas of $I^{\mathfrak{b}_{0}}(\lambda)$. If $\lambda$ is an atypical weight, then in view of $\operatorname{Remark} 3.4$, we have $\operatorname{mult}(\lambda, \nu)=0$ 
for all typical weights $\nu$. Therefore, it is sufficient to $\operatorname{discuss} \operatorname{mult}(\lambda, \mu)$, where $\lambda, \mu$ are atypical weights.

The following lemma is straightforward.

Lemma 3.5. Let $\lambda, \mu$ be atypical weights.

(1) Suppose $\operatorname{len}\left(L^{\mathfrak{b}_{0}}(\mu)\right)=p n-1$. If $\operatorname{mult}(\lambda, \mu) \neq 0$, then $\mu=\lambda$ or $\mu^{\prime}=\lambda-2 \sum_{i=1}^{n} \varepsilon_{i}$.

(2) Suppose $\operatorname{len}\left(L^{\mathfrak{b}_{0}}(\mu)\right)=p n-2$. If $\operatorname{mult}(\lambda, \mu) \neq 0$, then either $\mu=\lambda, \lambda-\varepsilon_{k}-\delta$, $\lambda+\varepsilon_{k}-\delta$ for some $k$ with $1 \leq k \leq n$ or $\mu^{\prime}=\lambda-2 \sum_{i=1}^{n} \varepsilon_{i}$.

Let $\lambda, \mu$ be atypical weights. By Lemma 3.3, when $\mu \in\left\{a \delta, \varepsilon_{n, 1, a} \mid a \in \mathbb{F}_{p}\right\}$, we have $\operatorname{len}\left(L^{\mathfrak{b}_{0}}(\mu)\right)<p n-1$. In this situation, it is not easy to determine whether $L^{\mathfrak{b}_{0}}(\mu)$ is a composition factor of $I^{\mathfrak{b}_{0}}(\lambda)$. Therefore we establish the following two lemmas to treat this special case.

Lemma 3.6. Let $\lambda$ be any atypical weight and $\mu=a \delta$, where $a \in \mathbb{F}_{p}$. Then $\operatorname{mult}(\lambda, \mu) \neq 0$ if and only if one of the following statements hold:

(1) $\lambda=\varepsilon_{i, 1, a}$ for some $i$ with $1 \leq i \leq n$;

(2) $\lambda=\varepsilon_{i, a}$ for some $i$ with $1 \leq i \leq n$.

Proof. As $\mathfrak{g}_{[0]}$-modules, we have $I^{\mathfrak{b}_{0}}(\lambda) \cong L^{0}(\lambda) \otimes_{\mathfrak{g}_{[0]}} \mathbf{u}\left(\mathfrak{g}_{[-1]}\right)$. Therefore,

$$
\operatorname{Hom}_{\mathfrak{g}_{[0]}}\left(L^{\mathfrak{b}_{0}}(\lambda), I^{\mathfrak{b}_{0}}(\mu)\right) \cong \operatorname{Hom}_{\mathfrak{g}_{[0]}}\left(L^{0}(\lambda) \otimes_{\mathfrak{g}_{[0]}} \mathbf{u}\left(\mathfrak{g}_{[-1]}\right), L^{\mathfrak{b}_{0}}(\mu)\right)
$$

Note that for a Lie superalgebra $L$ and $L$-modules $V, W$ and $N$,

$$
V^{*} \bigotimes_{L} W \cong \operatorname{Hom}_{L}(V, W)
$$

and

$$
\operatorname{Hom}_{L}\left(V, \operatorname{Hom}_{L}(W, N)\right) \cong \operatorname{Hom}_{L}\left(V \bigotimes_{L} W, N\right)
$$

Then we have

$$
\operatorname{Hom}_{\mathfrak{g}_{[0]}}\left(L^{0}(\lambda) \bigotimes_{\mathfrak{g}_{[0]}} \mathbf{u}\left(\mathfrak{g}_{[-1]}\right), L^{\mathfrak{b}_{0}}(\mu)\right) \cong \operatorname{Hom}_{\mathfrak{g}_{[0]}}\left(\mathbf{u}\left(\mathfrak{g}_{[-1]}\right), L^{\mathfrak{b}_{0}}(\mu) \bigotimes_{\mathfrak{g}_{[0]}}\left(L^{0}(\lambda)\right)^{*}\right)
$$

and

$$
\operatorname{Hom}_{\mathfrak{g}_{[0]}}\left(I^{\mathfrak{b}_{0}}(\lambda), L^{\mathfrak{b}_{0}}(\mu)\right) \cong I^{\mathfrak{b}_{0}}(\lambda)^{*} \bigotimes_{\mathfrak{g}_{[0]}} L^{\mathfrak{b}_{0}}(\mu)
$$


To consider the necessity, suppose $\operatorname{mult}(\lambda, \mu) \neq 0$. Then by $(3.3)$, we have $I^{\mathfrak{b}_{0}}(\lambda)^{*} \bigotimes_{\mathfrak{g}_{[0]}}$ $L^{\mathfrak{b}_{0}}(\mu) \neq 0$. It follows from $(3.1), 3.2$ and 3.3 that

$$
\operatorname{Hom}_{\mathfrak{g}_{[0]}}\left(\mathbf{u}\left(\mathfrak{g}_{[-1]}\right), L^{\mathfrak{b}_{0}}(\mu) \bigotimes_{\mathfrak{g}_{[0]}}\left(L^{0}(\lambda)\right)^{*}\right) \neq 0
$$

Since $\mu \in \mathbb{F}_{p} \delta$, we have $\operatorname{dim} L^{\mathfrak{b}_{0}}(\mu)=1$ and then $L^{\mathfrak{b}_{0}}(\mu) \bigotimes_{\mathfrak{g}_{[0]}}\left(L^{0}(\lambda)\right)^{*} \cong L^{\mathfrak{b}_{0}}(\mu-\lambda)$. Hence

$$
\operatorname{Hom}_{\mathfrak{g}_{[0]}}\left(\mathbf{u}\left(\mathfrak{g}_{[-1]}\right), L^{\mathfrak{b}_{0}}(b \delta) \bigotimes_{\mathfrak{g}_{[0]}}\left(L^{0}(\lambda)\right)^{*}\right) \cong \operatorname{Hom}_{\mathfrak{g}_{[0]}}\left(\mathbf{u}\left(\mathfrak{g}_{[-1]}\right), L^{0}(\mu-\lambda)\right) \neq 0
$$

It follows that $\mathfrak{g}_{[0]}$-module $\mathbf{u}\left(\mathfrak{g}_{[-1]}\right)$ contains a highest weight vector, denoted by $v_{\mu-\lambda}$, of weight $\mu-\lambda$ with respect to $\mathfrak{b}_{[0]}$. Since $v_{\mu-\lambda} \otimes v_{\lambda}$ is a highest weight vector in $\mathbf{u}\left(\mathfrak{g}_{[-1]}\right) \bigotimes_{\mathfrak{g}_{[0]}} L^{0}(\lambda)$ of weight $\mu$ with respect to $\mathfrak{b}_{[0]}$ and

$$
I^{\mathfrak{b}_{0}}(\lambda) \cong \mathbf{u}\left(\mathfrak{g}_{[-1]}\right) \bigotimes_{\mathfrak{g}_{[0]}} L^{0}(\lambda)
$$

one sees that $I^{\mathfrak{b}_{0}}(\lambda)$ contains a highest weight vector of weight $\mu$. Moreover, all weights of $\mathfrak{g}_{[0]}$-module $\mathbf{u}\left(\mathfrak{g}_{[-1]}\right)$ are of the form:

$$
\left(-\varepsilon_{i_{1}}-\delta\right)+\cdots+\left(-\varepsilon_{i_{k}}-\delta\right)+r_{1}\left(\varepsilon_{j_{1}}-\delta\right)+\cdots+r_{l}\left(\varepsilon_{j_{l}}-\delta\right)
$$

where $1 \leq i_{1}<\cdots<i_{k} \leq n, 1 \leq j_{1}<\cdots<j_{l} \leq n$ and $0 \leq r_{i} \leq p-1$. Then we have

$$
\mu=\lambda+\left(-\varepsilon_{i_{1}}-\delta\right)+\cdots+\left(-\varepsilon_{i_{k}}-\delta\right)+r_{1}\left(\varepsilon_{j_{1}}-\delta\right)+\cdots+r_{l}\left(\varepsilon_{j_{l}}-\delta\right)
$$

Since $\lambda$ is atypical, it follows that

$$
\lambda=\varepsilon_{i, 1, a} \quad \text { or } \quad \varepsilon_{i, a}
$$

for some $a \in \mathbb{F}_{p}$ and some $i$ with $1 \leq i \leq n$.

Suppose $\lambda=\varepsilon_{i, 1, a}$, where $a \in \mathbb{F}_{p}$ and $1 \leq i \leq n$. Note that the natural $\mathbb{Z}$-grading of $\mathbf{u}\left(\mathfrak{g}_{[-1]}\right)$ :

$$
\mathbf{u}\left(\mathfrak{g}_{[-1]}\right)=\bigoplus_{i=0}^{p n} \mathbf{u}\left(\mathfrak{g}_{[-1]}\right)_{i}
$$

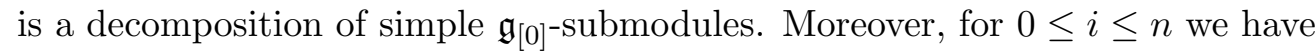

$$
\mathbf{u}\left(\mathfrak{g}_{[-1]}\right)_{i} \cong L^{0}\left(-\sum_{j=1}^{i} \varepsilon_{j}-i \delta\right)
$$


and for $n+1 \leq i \leq p n$ we have

$$
\mathbf{u}\left(\mathfrak{g}_{[-1]}\right)_{i} \cong L^{0}\left(-\sum_{j=1}^{n} \varepsilon_{j}-\sum_{j=k+1}^{n} \varepsilon_{j}+l \varepsilon_{k}-(k+l) \delta\right),
$$

where $1 \leq k \leq n$ and $0 \leq l \leq p-1$ with $n+l+(p-1)(n-k)=i$. Since $I^{\mathfrak{b}_{0}}(\lambda)$ contains a highest weight vector of weight $\mu$, it follows from (3.4) and (3.5) that $\mu=a \delta$.

Now suppose $\lambda=\varepsilon_{i, a}$, where $a \in \mathbb{F}_{p}$ and $1 \leq i \leq n$. Then, completely analogous to the arguments in the situation $\lambda=\varepsilon_{i, 1, a}$, one may obtain that $\mu=a \delta$.

Finally, let us consider the sufficiency. Suppose (1) or (2) holds, say, (1). By a direct verification, we get that $\operatorname{De}_{x_{i}} \cdots \mathrm{De}_{x_{1}} v_{\lambda}$ is a highest weight vector in $I^{\mathfrak{b}_{0}}(\lambda)$ of weight $\mu=a \delta$ with respect to $\mathfrak{b}_{i}$. Since $L^{\mathfrak{b}_{i}}(\mu) \cong L^{\mathfrak{b}_{0}}(\mu)$, we have $\operatorname{mult}(\lambda, \mu) \neq 0$.

Lemma 3.7. Let $\lambda$ be any atypical weight and $\mu=\varepsilon_{n, 1, a}$, where $a \in \mathbb{F}_{p}$. Then $\operatorname{mult}(\lambda, \mu) \neq$ 0 if and only if $\lambda$ is one of the following weights

$$
\mu, \mu+2 \delta, \mu-\varepsilon_{n}+\delta, \mu+\varepsilon_{n}+\delta .
$$

Proof. Suppose mult $(\lambda, \mu) \neq 0$. By Lemmas 3.1, 3.3 and 3.5, $\lambda$ must be one of the following weights

$$
\mu, \mu+2 \delta, \mu-\varepsilon_{n}+\delta \text { or } \mu+\varepsilon_{n}+\delta .
$$

Conversely, it easy to see that $\operatorname{mult}(\mu, \mu) \neq 0$ and $\operatorname{mult}(\mu+2 \delta, \mu) \neq 0$. Let $\lambda \in \Omega$ and $v_{\lambda}$ be a highest weight vector in $I^{\mathfrak{b}_{0}}(\lambda)$ of weight $\lambda$ with respect to $\mathfrak{b}_{0}$. If $\lambda=\mu+\varepsilon_{n}+\delta$, then

$$
\operatorname{De}_{x_{1^{\prime}} x_{n} x_{n^{\prime}}} \cdots \operatorname{De}_{x_{(n-1)^{\prime}} x_{n} x_{n^{\prime}}} \operatorname{De}_{x_{n}} \cdots \operatorname{De}_{x_{1}} \cdot v_{\lambda}
$$

is a highest weight vector in $I^{\mathfrak{b}_{0}}(\lambda)$ of weight $\mu$ with respect to $\mathfrak{b}_{0}$. If $\lambda=\mu-\varepsilon_{n}+\delta$, then

$$
\operatorname{De}_{x_{1^{\prime}} x_{n} x_{n^{\prime}}} \cdots \mathrm{De}_{x_{(n-1)^{\prime}} x_{n} x_{n^{\prime}}} \mathrm{De}_{x_{n^{\prime}} x_{n-1} x_{(n-1)^{\prime}}} \mathrm{De}_{x_{n-1}} \cdots \mathrm{De}_{x_{1}} \cdot v_{\lambda}
$$

is a highest weight vector in $I^{\mathfrak{b}_{0}}(\lambda)$ of weight $\mu$ with respect to $\mathfrak{b}_{0}$. Thus $\operatorname{mult}(\lambda, \mu) \neq 0$.

By PBW theorem, any element $v \in I^{\mathfrak{b}_{0}}(\lambda)$ can be uniquely written in the form

$$
v=\sum_{s \in \mathbf{I}(n, \underline{1} \mid n)} \operatorname{De}^{s} \otimes v(s)
$$

where $v(s) \in L^{0}(\lambda)$ and

$$
\mathrm{De}^{s}=\mathrm{De}_{x_{1^{\prime}}}^{s_{1}} \cdots \mathrm{De}_{x_{n^{\prime}}}^{s_{n}} \mathrm{De}_{x_{(n+1)^{\prime}}}^{s_{n+1}} \cdots \mathrm{De}_{x_{(2 n)^{\prime}}}^{s_{2 n}} .
$$

We conclude this section by establishing the following lemma, which will be used in the next section to determine mult $(\lambda, \mu)$ for atypical weights (see Proposition 4.1). 
Lemma 3.8. Let $v=\sum_{s \in \mathbf{I}(n, \underline{1} \mid n)} \operatorname{De}^{s} \otimes v(s)$ be a highest weight vector in $I^{\mathfrak{b}_{0}}(\lambda)$ of weight a $\delta$ with respect to $\mathfrak{b}_{[0]}$, where $a \in \mathbb{F}_{p}$. If $v(s) \neq 0$ for some $s \in \mathbf{I}(n, \underline{1} \mid n)$, then the following statements hold:

(1) if $s_{i^{\prime}}=1$, then $s_{i} \in\{0, p-1\}$, where $1 \leq i \leq n$;

(2) if $s_{i^{\prime}}=0$, then $s_{i} \in\{0,1, p-1\}$, where $1 \leq i \leq n$;

(3) if $s_{i}=p-1$ for some $1 \leq i \leq n$, then $s=(p-1, \ldots, p-1 \mid 1, \ldots, 1)$;

(4) if $s_{i}=1$ for some $1 \leq i \leq n$, then $s=\epsilon_{i}$.

Proof. For each $1 \leq i \leq n$, we have

$$
0=\operatorname{De}_{x_{i} x_{i^{\prime}}} \cdot v=\sum_{s \in \mathbf{I}(n, \underline{1} \mid n)} \operatorname{De}^{s} \otimes\left(\operatorname{De}_{x_{i} x_{i^{\prime}}} \cdot v(s)+\left(s_{i}-s_{i^{\prime}}\right) v(s)\right) .
$$

Therefore, $\operatorname{De}_{x_{i} x_{i^{\prime}}} \cdot v(s)=\left(s_{i^{\prime}}-s_{i}\right) v(s)$.

(1) For each $1 \leq i \leq n$, we have

$$
\begin{aligned}
0=\operatorname{De}_{x^{\left(2 \epsilon_{i}+\epsilon_{i^{\prime}}\right)} \cdot v=} & -\sum_{s \in \mathbf{I}(n, \underline{1} \mid n)} s_{i^{\prime}} \mathrm{De}^{s-\epsilon_{i^{\prime}}} \otimes \operatorname{De}_{x^{\left(2 \epsilon_{i}\right)} \cdot v(s)} \\
& -\sum_{s \in \mathbf{I}(n, \underline{1} \mid n)} \frac{s_{i}\left(s_{i}+1\right)}{2} \mathrm{De}^{s-\epsilon_{i}} \otimes v(s) .
\end{aligned}
$$

If $s_{i^{\prime}}=1$, then the term $\frac{s_{i}\left(s_{i}+1\right)}{2} \mathrm{De}^{s-\epsilon_{i^{\prime}}} \otimes v(s)$ in 3.7 does not cancel with any other terms in (3.6) and (3.7). It follows that $s_{i}=0$ or $p-1$.

(2) Suppose $s_{i} \notin\{0, p-1\}$. Then the term $\frac{s_{i}\left(s_{i}+1\right)}{2} \mathrm{De}^{s-\epsilon_{i}} \otimes v(s)$ in 3.7 is nonzero and

$$
\sum_{s \in \mathbf{I}(n, \underline{1} \mid n)} \operatorname{De}^{s-\epsilon_{i}} \otimes \operatorname{De}_{x^{\left(2 \epsilon_{i}\right)} \cdot v\left(s-\epsilon_{i}+\epsilon_{i^{\prime}}\right)}
$$

in 3.6 is nonzero. By (1), we have $s_{i}-1 \in\{0, p-1\}$. Then $s_{i}=1$.

(3) If $1 \leq i \neq j \leq n$, we have

$$
\begin{aligned}
0=\operatorname{De}_{x^{\left(2 \epsilon_{i}+\epsilon_{j^{\prime}}\right)} \cdot v=} & -\sum_{s \in \mathbf{I}(n, \underline{1} \mid n)} s_{j^{\prime}} \mathrm{De}^{s-\epsilon_{j^{\prime}}} \otimes \mathrm{De}_{x^{\left(2 \epsilon_{i}\right)}} \cdot v(s) \\
& +\sum_{s \in \mathbf{I}(n, \underline{1} \mid n)} s_{i} \mathrm{De}^{s-\epsilon_{i}} \otimes \mathrm{De}_{x_{i} x_{j^{\prime}}} \cdot v(s) \\
& +\sum_{s \in \mathbf{I}(n, \underline{1} \mid n)} \frac{s_{i}\left(s_{i}-1\right)}{2} \mathrm{De}^{s-2 \epsilon_{i}+\epsilon_{j}} \otimes v(s) \\
& -\sum_{s \in \mathbf{I}(n, \underline{1} \mid n)} s_{i} \mathrm{De}^{s-\epsilon_{i}-\epsilon_{j^{\prime}}+\epsilon_{i^{\prime}}} \otimes v(s) .
\end{aligned}
$$


Suppose $s_{i}=p-1, s_{j} \neq p-1$ for some $i$ and $j$ with $1 \leq i \neq j \leq n$. Then by (1) and (2), the term $\frac{(p-1)(p-2)}{2} \mathrm{De}^{s-2 \epsilon_{i}+\epsilon_{j}} \otimes v(s)$ in the third sum of the right-hand side of 3.8 does not cancel with any other terms in $(3.8)$. Hence $v(s)=0$. So far, we have shown that if $v(s) \neq 0$ and $s_{i}=p-1$ for some $i$ with $1 \leq i \leq n$, then $s_{j}=p-1$ for all $j$ with $1 \leq j \leq n$. If $1 \leq i \neq j \leq n$, we have

$$
\begin{aligned}
0=\operatorname{De}_{x_{i} x_{j}} \cdot v= & \sum_{s \in \mathbf{I}(n, \underline{1} \mid n)} \operatorname{De}^{s} \otimes \operatorname{De}_{x_{i} x_{j}} \cdot v(s) \\
& +\sum_{s \in \mathbf{I}(n, \underline{1} \mid n)} s_{j} \operatorname{De}^{s-\epsilon_{j}+\epsilon_{i^{\prime}}} \otimes v(s) \\
& +\sum_{s \in \mathbf{I}(n, \underline{1} \mid n)} s_{i} \operatorname{De}^{s-\epsilon_{i}+\epsilon_{j^{\prime}}} \otimes v(s) .
\end{aligned}
$$

Suppose $s_{i}=p-1, s_{j^{\prime}}=0$ with $1 \leq i \neq j \leq n$. Then by (1) and (2), the term $s_{i} \mathrm{De}^{s-\epsilon_{i}+\epsilon_{j^{\prime}}} \otimes v(s)$ in the third sum of the right-hand side of $(3.9)$ does not cancel with any other terms in $(3.9)$. Hence $v(s)=0$. So far, we have shown that if $v(s) \neq 0$ and $s_{i}=p-1$ for some $i$ with $1 \leq i \leq n$, then $s_{j^{\prime}}=1$ for all $j$ with $1 \leq j \leq n$.

(4) By (1), (2) and (3), we have $s_{1}, \ldots, s_{n} \in\{0,1\}$, when $v(s) \neq 0$ and $s_{i}=1$ for some $i$ with $1 \leq i \leq n$. If $1 \leq i<j \leq n$, we have

$$
\begin{aligned}
0=\operatorname{De}_{x_{i} x_{j^{\prime}}} \cdot v= & \sum_{s \in \mathbf{I}(n, \underline{1} \mid n)} \operatorname{De}^{s} \otimes \operatorname{De}_{x_{i} x_{j^{\prime}}} \cdot v(s) \\
& -\sum_{s \in \mathbf{I}(n, \underline{1} \mid n)} s_{j^{\prime}} \operatorname{De}^{s-\epsilon_{j^{\prime}}+\epsilon_{i^{\prime}}} \otimes v(s) \\
& +\sum_{s \in \mathbf{I}(n, \underline{1} \mid n)} s_{i} \operatorname{De}^{s-\epsilon_{i}+\epsilon_{j}} \otimes v(s)
\end{aligned}
$$

Suppose $s_{i}=1, s_{j}=1$ for some $i$ and $j$ with $1 \leq i<j \leq n$. Then by (1) and (2), the term $s_{i} \mathrm{De}^{s-\epsilon_{i}+\epsilon_{j}} \otimes v(s)$ in the third sum of the right-hand side of $(3.10)$ does not cancel with any other terms in 3.10 . Hence $v(s)=0$. Suppose $s_{i}=1, s_{i^{\prime}}=1$ for some $i$ with $1 \leq i \leq n$. Then the term $\operatorname{De}^{s-\epsilon_{i}} \otimes v(s)$ in (3.7) does not cancel with any other terms in (3.6) and (3.7). Hence $v(s)=0$. Suppose $s_{i}=1, s_{j^{\prime}}=1$ for some $i$ and $j$ with $1 \leq i \neq j \leq n$. Then the term $\mathrm{De}^{s-\epsilon_{i}+\epsilon_{j}} \otimes v(s)$ in the third sum of the right-hand side of (3.10) does not cancel with any other terms in 3.10. Hence $v(s)=0$. So far, we have shown that $s=\epsilon_{i}$, when $v(s) \neq 0$ and $s_{i}=1$ for some $i$ with $1 \leq i \leq n$.

\section{Character formulas}

We first establish several propositions, which will be used in determining the character formulas. 
Proposition 4.1. Let $\lambda, \mu$ be atypical weights. Then $\operatorname{mult}(\lambda, \mu) \leq 1$.

Proof. One may suppose $\operatorname{mult}(\lambda, \mu) \neq 0$. Then $L^{\mathfrak{b}_{0}}(\mu)$ is a subquotient of $I^{\mathfrak{b}_{0}}(\lambda)$, that is, there exist submodules $M$ and $N$ of $I^{\mathfrak{b}_{0}}(\lambda)$ with $M \supseteq N$ such that $M / N \cong L^{\mathfrak{b}_{0}}(\mu)$. Let $v \in M \subseteq I^{\mathfrak{b}_{0}}(\lambda)$ be an inverse image of some highest weight vector in $L^{\mathfrak{b}_{0}}(\mu)$ of weight $\mu$ under the canonical homomorphism. Our discussion is divided into two parts.

Part 1: Suppose $\mu \notin \mathbb{F}_{p} \delta$. One may write $L^{\mathfrak{b}_{0}}(\mu)=\bigoplus_{i=l}^{h} L^{\mathfrak{b}_{0}}(\mu)_{i}$ and $\lambda=\sum_{i=1}^{n} a_{i} \varepsilon_{i}+$ $a \delta$, where $a_{i}, a \in \mathbb{F}_{p}$. Note that any nonzero submodule of $I^{\mathfrak{b}_{0}}(\lambda)$ contains $\operatorname{De}_{x_{1^{\prime}}}^{p-1} \cdots \operatorname{De}_{x_{n^{\prime}}}^{p-1}$ $\operatorname{De}_{x_{n}} \cdots \operatorname{De}_{x_{1}} v_{\lambda}$. Hence $L^{\mathfrak{b}_{0}}(\mu)_{a-p n}=0$. By Lemma 3.3 , we have $\operatorname{len}\left(L^{\mathfrak{b}_{0}}(\mu)\right)=p n-1$ or $p n-2$. Then $h \in\{a, a-1\}$. Therefore $v \in I^{\mathfrak{b}_{0}}(\lambda)_{a}$ or $I^{\mathfrak{b}_{0}}(\lambda)_{a-1}$, that is, $v \in$ $\mathbf{u}\left(\mathfrak{g}_{[-1]}\right)_{0} \otimes L^{0}(\lambda)$ or $v \in \mathbf{u}\left(\mathfrak{g}_{[-1]}\right)_{1} \otimes L^{0}(\lambda)$. If $v \in \mathbf{u}\left(\mathfrak{g}_{[-1]}\right)_{0} \otimes L^{0}(\lambda)$, then $M=I^{\mathfrak{b}_{0}}(\lambda)$ and $\mu=\lambda$, and hence $v$ is a highest weight vector in $I^{\mathfrak{b}_{0}}(\lambda)$ of weight $\mu$. Suppose $v \in \mathbf{u}\left(\mathfrak{g}_{[-1]}\right)_{1} \otimes L^{0}(\lambda)$. On the one hand, $\mathfrak{u}\left(\mathfrak{b}_{0}\right) v \subseteq \bigoplus_{j \leq 1} \mathbf{u}\left(\mathfrak{g}_{[-1]}\right)_{j} \otimes L^{0}(\lambda)$ from $\mathfrak{u}\left(\mathfrak{b}_{0}\right) \bar{v} \in \mathbb{F} \bar{v}$. On the other hand, $\mathbf{u}\left(\mathfrak{b}_{0}\right) I^{\mathfrak{b}_{0}}(\lambda)_{1} \subseteq \bigoplus_{j \geq 1} I^{\mathfrak{b}_{0}}(\lambda)_{j}$, one may prove that $v$ is a highest weight vector in $I^{\mathfrak{b}_{0}}(\lambda)$ of weight $\mu$. As in the proof of [2, Theorem 3.6], we can prove that the dimension of the space spanned by highest weight vectors in $\bigoplus_{i=0}^{1} \mathbf{u}\left(\mathfrak{g}_{[-1]}\right)_{i} \otimes L^{0}(\lambda)$ of weight $\mu$ is not bigger than the multiplicity of weight $\mu-\lambda$ in $\bigoplus_{i=0}^{1} \mathbf{u}\left(\mathfrak{g}_{[-1]}\right)_{i}$. Note that the multiplicity of each weight in $\bigoplus_{i=0}^{1} \mathbf{u}\left(\mathfrak{g}_{[-1]}\right)_{i}$ is 1 . Therefore, $\operatorname{mult}(\lambda, \mu) \leq 1$.

Part 2: Suppose $\mu \in \mathbb{F}_{p} \delta$, that is, $\mu=a \delta$ for some $a \in \mathbb{F}_{p}$. As in the proof of Lemma 3.6, one may see that there exists a highest weight vector of weight $\mu$ in $I^{\mathfrak{b}_{0}}(\lambda)$. Thus one may assume that $v$ is such a highest weight vector. Then it follows from Lemma 3.8 that

$$
v \in\left(\mathbf{u}\left(\mathfrak{g}_{[-1]}\right)_{p n} \oplus \mathbf{u}\left(\mathfrak{g}_{[-1]} \cap \mathfrak{g}_{\overline{0}}\right)_{1} \oplus \mathbf{u}\left(\mathfrak{g}_{[-1]} \cap \mathfrak{g}_{\overline{1}}\right)\right) \otimes L^{0}(\lambda)
$$

Note that the multiplicity is 1 for each weight in

$$
\mathbf{u}\left(\mathfrak{g}_{[-1]}\right)_{p n} \oplus \mathbf{u}\left(\mathfrak{g}_{[-1]} \cap \mathfrak{g}_{\overline{0}}\right)_{1} \oplus \mathbf{u}\left(\mathfrak{g}_{[-1]} \cap \mathfrak{g}_{\overline{1}}\right)
$$

Then, similar to Part 1 , we have $\operatorname{mult}(\lambda, \mu) \leq 1$. The proof is complete.

Proposition 4.2. Let $\lambda, \mu$ be atypical weights. Then $\operatorname{mult}(\lambda, \mu) \neq 0$ if and only if $\operatorname{mult}(\lambda, \mu)=1$. In this case, $(\lambda, \mu)$ is exactly of one of the following forms for some $a \in \mathbb{F}_{p}$ :

(1) $\lambda=a \delta, \mu=a \delta$ or $\varepsilon_{1,-1, a}$.

(2) $\lambda=\varepsilon_{1, a}, \mu=\lambda$ or $a \delta$.

(3) $\lambda=\varepsilon_{i, a}, \mu=\lambda$, a $\delta$ or $\varepsilon_{i-1, a}$ for some $2 \leq i \leq n-1$.

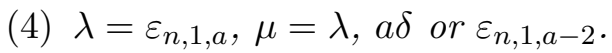


(5) $\lambda=\varepsilon_{n, a}, \mu=\lambda, \varepsilon_{n-1, a}$ or $\varepsilon_{n, 1, a-2}$

(6) $\lambda=\varepsilon_{n, a, b}, \mu=\lambda$ or $\varepsilon_{n, a-1, b}$ with $a \neq 0,1,2$.

(7) $\lambda=\varepsilon_{n-1,1, a}, \mu=\lambda, \varepsilon_{n,-1, a}$, a $\delta$ or $\varepsilon_{n, 1, a-2}$.

(8) $\lambda=\varepsilon_{i, 1, a}, \mu=\lambda$, a or $\varepsilon_{i+1,-1, a}$ for some $1 \leq i \leq n-2$.

(9) $\lambda=\varepsilon_{i, a, b}, \mu=\lambda$ or $\varepsilon_{i, a-1, b}$ for some $1 \leq i \leq n-1, a \neq 0,1$.

Proof. The first conclusion follows directly from Proposition 4.1. By the definition of an atypical weight, $\lambda$ must be of one of the forms (1)-(9) indicated above.

(1) By Lemma 3.7, we have $\mu \notin \sum_{j=1}^{n} \varepsilon_{j}+\mathbb{F}_{p} \delta$. If $\mu \notin \mathbb{F}_{p} \delta$, then by Lemma 3.3, we have len $\left(L^{\mathfrak{b}_{0}}(\mu)\right)=n p-1$. By Lemma 3.5, we have $\mu^{\prime}=a \delta-2 \sum_{i=1}^{n} \varepsilon_{i}$. By Lemma 3.1. we have $\mu=\varepsilon_{1,-1, a}$. Then $L^{\mathfrak{b}_{0}}\left(\varepsilon_{1,-1, a}\right)$ is the minimal submodule of $I^{\mathfrak{b}_{0}}(a \delta)$. If $\mu \in \mathbb{F}_{p} \delta$, then by Lemma 3.6 we have $\mu=a \delta$.

(2) Obviously, $L^{\mathfrak{b}_{0}}(a \delta)$ is the minimal submodule of $I^{\mathfrak{b}_{0}}(\lambda)$. By Lemma 3.7, we have $\operatorname{len}\left(L^{\mathfrak{b}_{0}}(\mu)\right) \neq p n-2$. By Lemma 3.5, we have $\mu=\lambda$ or $a \delta$.

(3) If $\mu \notin \mathbb{F}_{p} \delta$, by Lemma 3.7. we have $\operatorname{len}\left(L^{\mathfrak{b}_{0}}(\mu)\right)=p n-1$. Then by Lemmas 3.1 and 3.5, we have $\mu=\lambda$ or $\mu=\varepsilon_{i-1, a}$. If $\mu \in \mathbb{F}_{p} \delta$, then by Lemma 3.6, we have $\mu=a \delta$.

(4) We claim that $\operatorname{mult}(\lambda, \mu)=0$ if $\operatorname{len}\left(L^{\mathfrak{b}_{0}}(\mu)\right)=p n-1$. Suppose len $\left(L^{\mathfrak{b}_{0}}(\mu)\right)=p n-1$ and $\operatorname{mult}(\lambda, \mu) \neq 0$. Then by Lemma 3.5, we have $\mu^{\prime}=\lambda-2 \sum_{i=1}^{n} \varepsilon_{i}$. By Lemma 3.1, we have $\mu=\varepsilon_{n, 1, a-2}$, which contradicts the assumption on $\mu$. Hence our claim is true. Then $\operatorname{len}\left(L^{\mathfrak{b}_{0}}(\mu)\right)=p n-2$ or $\mu \in \mathbb{F}_{p} \delta$. By Lemmas 3.1 3.5, we have $\mu=\lambda, \varepsilon_{n, 1, a-2}$ or $a \delta$.

(5) By Lemma 3.6, we have $\mu \notin \mathbb{F}_{p} \delta$. Then by Lemma 3.3, we may assume that $\operatorname{len}\left(L^{\mathfrak{b}_{0}}(\mu)\right)=p n-1$ or $p n-2$. If $\operatorname{len}\left(L^{\mathfrak{b}_{0}}(\mu)\right)=p n-1$, then by Lemmas 3.1 and 3.5, we have $\mu=\lambda$ or $\varepsilon_{n-1, a}$. If $\operatorname{len}\left(L^{\mathfrak{b}_{0}}(\mu)\right)=p n-2$, then by Lemma 3.7 , we have $\mu=\varepsilon_{n, 1, a-2}$.

(6) By Lemma 3.6, we have $\mu \notin \mathbb{F}_{p} \delta$. Then by Lemmas 3.3 and 3.7, we may assume that $\operatorname{len}\left(L^{\mathfrak{b}_{0}}(\mu)\right)=p n-1$. By Lemmas 3.1 and 3.5, we have $\mu=\lambda$ or $\varepsilon_{n, a-1, b}$.

(7) Let $L^{\mathfrak{b}_{0}}(\mu)$ be the minimal submodule of $I^{\mathfrak{b}_{0}}(\lambda)$. Then $\mu^{\prime}=\lambda+\sigma$. So $\mu=\varepsilon_{n,-1, a}$. We have the following exact sequences

$$
0 \longrightarrow M \longrightarrow I^{\mathfrak{b}_{0}}(\lambda) \longrightarrow L^{\mathfrak{b}_{0}}(\lambda) \longrightarrow 0
$$

and

$$
0 \longrightarrow L^{\mathfrak{b}_{0}}\left(\varepsilon_{n,-1, a}\right) \longrightarrow M \longrightarrow N(\lambda) \longrightarrow 0
$$

where $M$ is the maximal submodule of $I^{\mathfrak{b}_{0}}(\lambda)$ and $N(\lambda)$ is the quotient of the maximal submodule of $I^{\mathfrak{b}_{0}}(\lambda)$ modulo the minimal submodule. Next, we consider the structure of $N(\lambda)$. Let $L^{\mathfrak{b}_{0}}(\nu)$ be a subquotient of $N(\lambda)$. Since len $(N(\lambda)) \leq p n-2$, we get that $\nu \in \sum_{j=1}^{n} \varepsilon_{j}+\mathbb{F}_{p} \delta$ or $\nu \in \mathbb{F}_{p} \delta$. By Lemmas 3.6 and 3.7, we have $\operatorname{mult}(\lambda, a \delta) \neq 0$ and $\operatorname{mult}\left(\lambda, \varepsilon_{n, 1, a-2}\right) \neq 0$. 
Completely analogous to (3) and (6), one may verify (8) and (9), respectively.

By Proposition 4.2, we have the following corollary.

Corollary 4.3. Let $\lambda$ be an atypical weight and $M$ the maximal submodule of $I^{\mathfrak{b}_{0}}(\lambda)$.

(1) If $\lambda=a \delta$ with $a \in \mathbb{F}_{p}$, then the following sequence is exact:

$$
0 \longrightarrow L^{\mathfrak{b}_{0}}\left(\varepsilon_{1,-1, a}\right) \longrightarrow I^{\mathfrak{b}_{0}}(\lambda) \longrightarrow L^{\mathfrak{b}_{0}}(\lambda) \longrightarrow 0
$$

(2) If $\lambda=\varepsilon_{1, a}$ with $a \in \mathbb{F}_{p}$, then the following sequence is exact:

$$
0 \longrightarrow L^{\mathfrak{b}_{0}}(a \delta) \longrightarrow I^{\mathfrak{b}_{0}}(\lambda) \longrightarrow L^{\mathfrak{b}_{0}}(\lambda) \longrightarrow 0 .
$$

(3) If $\lambda=\varepsilon_{i, a}$ with $2 \leq i \leq n-1$ and $a \in \mathbb{F}_{p}$, then the following two sequences are exact:

$$
0 \longrightarrow M \longrightarrow I^{\mathfrak{b}_{0}}(\lambda) \longrightarrow L^{\mathfrak{b}_{0}}(\lambda) \longrightarrow 0
$$

and

$$
0 \longrightarrow L^{\mathfrak{b}_{0}}\left(\varepsilon_{i-1, a}\right) \longrightarrow M \longrightarrow L^{\mathfrak{b}_{0}}(a \delta) \longrightarrow 0
$$

(4) If $\lambda=\varepsilon_{n, 1, a}$ with $a \in \mathbb{F}_{p}$, then the following two sequences are exact:

$$
0 \longrightarrow M \longrightarrow I^{\mathfrak{b}_{0}}(\lambda) \longrightarrow L^{\mathfrak{b}_{0}}(\lambda) \longrightarrow 0
$$

and

$$
0 \longrightarrow L^{\mathfrak{b}_{0}}\left(\varepsilon_{n, 1, a-2}\right) \longrightarrow M \longrightarrow L^{\mathfrak{b}_{0}}(a \delta) \longrightarrow 0
$$

(5) If $\lambda=\varepsilon_{n, a}$ with $a \in \mathbb{F}_{p}$, then the following two sequences are exact:

$$
0 \longrightarrow M \longrightarrow I^{\mathfrak{b}_{0}}(\lambda) \longrightarrow L^{\mathfrak{b}_{0}}(\lambda) \longrightarrow 0
$$

and

$$
0 \longrightarrow L^{\mathfrak{b}_{0}}\left(\varepsilon_{n-1, a}\right) \longrightarrow M \longrightarrow L^{\mathfrak{b}_{0}}\left(\varepsilon_{n, 1, a-2}\right) \longrightarrow 0
$$

(6) If $\lambda=\varepsilon_{n, a, b}$ with $a, b \in \mathbb{F}_{p} \backslash\{0,1,2\}$, then the following sequence is exact:

$$
0 \longrightarrow L^{\mathfrak{b}_{0}}\left(\varepsilon_{n, a-1, b}\right) \longrightarrow I^{\mathfrak{b}_{0}}(\lambda) \longrightarrow L^{\mathfrak{b}_{0}}(\lambda) \longrightarrow 0 .
$$

(7) If $\lambda=\varepsilon_{n-1,1, a}$ with $a \in \mathbb{F}_{p}$, then the following two sequences are exact:

$$
0 \longrightarrow M \longrightarrow I^{\mathfrak{b}_{0}}(\lambda) \longrightarrow L^{\mathfrak{b}_{0}}(\lambda) \longrightarrow 0
$$

and

$$
0 \longrightarrow L^{\mathfrak{b}_{0}}\left(\varepsilon_{n,-1, a}\right) \longrightarrow M \longrightarrow L^{\mathfrak{b}_{0}}(a \delta) \oplus L^{\mathfrak{b}_{0}}\left(\varepsilon_{n, 1, a-2}\right) \longrightarrow 0
$$


(8) If $\lambda=\varepsilon_{i, 1, a}$ with $1 \leq i \leq n-2$ and $a \in \mathbb{F}_{p}$, then the following two sequences are exact:

$$
0 \longrightarrow M \longrightarrow I^{\mathfrak{b}_{0}}(\lambda) \longrightarrow L^{\mathfrak{b}_{0}}(\lambda) \longrightarrow 0
$$

and

$$
0 \longrightarrow L^{\mathfrak{b}_{0}}\left(\varepsilon_{i+1,-1, a}\right) \longrightarrow M \longrightarrow L^{\mathfrak{b}_{0}}(a \delta) \longrightarrow 0
$$

(9) If $\lambda=\varepsilon_{i, a, b}$ with $1 \leq i \leq n-1$ and $a, b \in \mathbb{F}_{p} \backslash\{0,1\}$, then the following sequence $i s$ exact:

$$
0 \longrightarrow L^{\mathfrak{b}_{0}}\left(\varepsilon_{i, a-1, b}\right) \longrightarrow I^{\mathfrak{b}_{0}}(\lambda) \longrightarrow L^{\mathfrak{b}_{0}}(\lambda) \longrightarrow 0
$$

Let $M=\bigoplus_{\mu \in \overline{\mathfrak{h}}^{*}} M_{\mu}$ be a $\mathfrak{g}$-module. Recall that the character of $M$ is

$$
\operatorname{ch} M=\sum_{\mu \in \overline{\mathfrak{h}}^{*}}\left(\operatorname{dim} M_{\mu}\right) e^{\mu}
$$

Let

$$
\Pi=\prod_{i=1}^{n}\left(1+e^{-\varepsilon_{i}-\delta}\right) \prod_{i=1}^{n}\left(1+e^{\varepsilon_{i}-\delta}\right)^{p-1} .
$$

Then $\operatorname{ch} I^{\mathfrak{b}_{0}}(\lambda)=\Pi \operatorname{ch} L^{0}(\lambda)$.

Suppose $0 \longrightarrow M^{\prime} \longrightarrow M \longrightarrow M^{\prime \prime} \longrightarrow 0$ is a short exact sequence, where $M^{\prime}$ and $M^{\prime \prime}$ are also weight modules of $\mathfrak{g}$. Since $\operatorname{dim} M_{\mu}=\operatorname{dim} M_{\mu}^{\prime}+\operatorname{dim} M_{\mu}^{\prime \prime}$ for any $\mu \in \overline{\mathfrak{h}}^{*}$, we have $\operatorname{ch} M=\operatorname{ch} M^{\prime}+\operatorname{ch} M^{\prime \prime}$. Thus $\operatorname{ch} M$ is determined by the characters and multiplicities of the composition factors of $M$. In particular, $\operatorname{ch} I^{\mathfrak{b}_{0}}(\lambda)=\sum_{\mu \in \overline{\mathfrak{h}}^{*}} \operatorname{mult}(\lambda, \mu) \operatorname{ch} L^{\mathfrak{b}_{0}}(\mu)$. Now we are in the position to prove the main result of this paper.

Theorem 4.4. Let $\lambda \in \mathbb{F}_{p}^{n+1}$. If $\lambda$ is typical, then $\operatorname{ch} L^{\mathfrak{b}_{0}}(\lambda)=\Pi \operatorname{ch} L^{0}(\lambda)$. If $\lambda$ is atypical, then $\lambda$ is of exactly one of the following nine forms and in each case the character formula is listed below:

(1) If $\lambda=a \delta$ with $a \in \mathbb{F}_{p}$, then $\operatorname{ch} L^{\mathfrak{b}_{0}}(\lambda)=e^{\lambda}$.

(2) If $\lambda=\varepsilon_{1, a}$ with $a \in \mathbb{F}_{p}$, then $\operatorname{ch} L^{\mathfrak{b}_{0}}(\lambda)=\Pi \operatorname{ch} L^{0}(\lambda)-e^{a \delta}$.

(3) If $\lambda=\varepsilon_{i, a}$ with $2 \leq i \leq n-1$ and $a \in \mathbb{F}_{p}$, then

$$
\operatorname{ch} L^{\mathfrak{b}_{0}}(\lambda)=\sum_{j=1}^{i}(-1)^{i-j} \Pi \operatorname{ch} L^{0}\left(\varepsilon_{j, a}\right)-\delta_{i+1 \in 2 \mathbb{Z}} e^{a \delta} .
$$

(4) If $\lambda=\varepsilon_{n, 1, a}$ with $a \in \mathbb{F}_{p}$, then

$$
\operatorname{ch} L^{\mathfrak{b}_{0}}(\lambda)=\frac{1}{2} \sum_{j=0}^{p-1}(-1)^{j}\left(\Pi \operatorname{ch} L^{0}\left(\varepsilon_{n, 1, a-2 j}\right)-e^{(a-2 j) \delta}\right) .
$$


(5) If $\lambda=\varepsilon_{n, a}$ with $a \in \mathbb{F}_{p}$, then

$$
\begin{aligned}
\operatorname{ch} L^{\mathfrak{b}_{0}}(\lambda)= & \sum_{j=1}^{n}(-1)^{n-j} \Pi \operatorname{ch} L^{0}\left(\varepsilon_{j, a}\right)+\delta_{n \in 2 \mathbb{Z}} e^{a \delta} \\
& -\frac{1}{2} \sum_{j=0}^{p-1}(-1)^{j}\left(\Pi \operatorname{ch} L^{0}\left(\varepsilon_{n, 1, a-2-2 j}\right)-e^{(a-2-2 j) \delta}\right) .
\end{aligned}
$$

(6) If $\lambda=\varepsilon_{n, a, b}$ with $a, b \in \mathbb{F}_{p} \backslash\{0,1,2\}$, then

$$
\begin{aligned}
\operatorname{ch} L^{\mathfrak{b}_{0}}(\lambda)= & \sum_{j=0}^{a-2}(-1)^{j} \Pi \operatorname{ch} L^{0}\left(\varepsilon_{n, a-j, b}\right)+\sum_{j=1}^{n}(-1)^{a+n-j} \Pi \operatorname{ch} L^{0}\left(\varepsilon_{j, b+2}\right) \\
& +(-1)^{a} \delta_{n \in 2 \mathbb{Z}} e^{(b+2) \delta}-\frac{1}{2} \sum_{j=0}^{p-1}(-1)^{a+j}\left(\Pi \operatorname{ch} L^{0}\left(\varepsilon_{n, 1, b-2 j}\right)-e^{(b-2 j) \delta}\right) .
\end{aligned}
$$

(7) If $\lambda=\varepsilon_{n-1,1, a}$ with $a \in \mathbb{F}_{p}$, then

$$
\begin{aligned}
\operatorname{ch} L^{\mathfrak{b}_{0}}(\lambda)= & \Pi \operatorname{ch} L^{0}(\lambda)-e^{a \delta}-\sum_{j=0}^{p-3}(-1)^{j} \Pi \operatorname{ch} L^{0}\left(\varepsilon_{n, p-1-j, a}\right) \\
& -\sum_{j=1}^{n}(-1)^{n-j} \Pi \operatorname{ch} L^{0}\left(\varepsilon_{j, a+2}\right)-\delta_{n \in 2 \mathbb{Z}} e^{(a+2) \delta} \\
& +\frac{1}{2} \sum_{j=0}^{p-1}(-1)^{j}\left(\Pi \operatorname{ch} L^{0}\left(\varepsilon_{n, 1, a-2 j}\right)-e^{(a-2 j) \delta}\right) \\
& -\frac{1}{2} \sum_{j=0}^{p-1}(-1)^{j}\left(\Pi \operatorname{ch} L^{0}\left(\varepsilon_{n, 1, a-2-2 j}\right)-e^{(a-2-2 j) \delta}\right) .
\end{aligned}
$$

(8) If $\lambda=\varepsilon_{i, 1, a}$ with $1 \leq i \leq n-2$ and $a \in \mathbb{F}_{p}$, then

$$
\begin{aligned}
\operatorname{ch} L^{\mathfrak{b}_{0}}(\lambda)= & \Pi \operatorname{ch} L^{0}(\lambda)+\sum_{j=1}^{p-1} \sum_{l=i+1}^{n-1}(-1)^{j} \Pi \operatorname{ch} L^{0}\left(\varepsilon_{l,-j, a}\right)-\Pi \operatorname{ch} L^{0}\left(\varepsilon_{n-1,1, a}\right) \\
& +\sum_{j=0}^{p-3}(-1)^{j} \Pi \operatorname{ch} L^{0}\left(\varepsilon_{n, p-1-j, a}\right)+\sum_{j=1}^{n}(-1)^{n-j} \Pi \operatorname{ch} L^{0}\left(\varepsilon_{j, a+2}\right) \\
& +\delta_{n \in 2 \mathbb{Z}} e^{(a+2) \delta}-\frac{1}{2} \sum_{j=0}^{p-1}(-1)^{j}\left(\Pi \operatorname{ch} L^{0}\left(\varepsilon_{n, 1, a-2 j}\right)-e^{(a-2 j) \delta}\right) \\
& +\frac{1}{2} \sum_{j=0}^{p-1}(-1)^{j}\left(\Pi \operatorname{ch} L^{0}\left(\varepsilon_{n, 1, a-2-2 j}\right)-e^{(a-2-2 j) \delta}\right)
\end{aligned}
$$


(9) If $\lambda=\varepsilon_{i, a, b}$ with $1 \leq i \leq n-1, a, b \in \mathbb{F}_{p}$ and $a \neq 0,1$, then

$$
\begin{aligned}
\operatorname{ch} L^{\mathfrak{b}_{0}}(\lambda)= & \left.\sum_{j=0}^{a-2}(-1)^{j} \Pi \operatorname{ch} L^{0}\left(\varepsilon_{i, a-j, b}\right)\right)+(-1)^{a} \Pi \operatorname{ch} L^{0}\left(\varepsilon_{i, 1, b}\right) \\
& +\sum_{j=1}^{p-1} \sum_{l=i+1}^{n-1}(-1)^{j+a} \Pi \operatorname{ch} L^{0}\left(\varepsilon_{l,-j, b}\right)-(-1)^{a} \Pi \operatorname{ch} L^{0}\left(\varepsilon_{n-1,1, b}\right) \\
& +\sum_{j=0}^{p-3}(-1)^{j+a} \Pi \operatorname{ch} L^{0}\left(\varepsilon_{n, p-1-j, b}\right)+\sum_{j=1}^{n}(-1)^{a+n-j} \Pi \operatorname{ch} L^{0}\left(\varepsilon_{j, b+2}\right) \\
& +(-1)^{a} \delta_{n \in 2 \mathbb{Z}} e^{(b+2) \delta}-\frac{1}{2} \sum_{j=0}^{p-1}(-1)^{a+j}\left(\Pi \operatorname{ch} L^{0}\left(\varepsilon_{n, 1, b-2 j}\right)-e^{(b-2 j) \delta}\right) \\
& +\frac{1}{2} \sum_{j=0}^{p-1}(-1)^{a+j}\left(\Pi \operatorname{ch} L^{0}\left(\varepsilon_{n, 1, b-2-2 j}\right)-e^{(b-2-2 j) \delta}\right) .
\end{aligned}
$$

Proof. If $\lambda$ is typical, by Lemma $3.2, I^{\mathfrak{b}_{0}}(\lambda)$ is simple and

$$
\operatorname{ch} L^{\mathfrak{b}_{0}}(\lambda)=\operatorname{ch} I^{\mathfrak{b}_{0}}(\lambda)=\Pi \operatorname{ch} L^{0}(\lambda)
$$

If $\lambda$ is atypical, by the definition of an atypical weight, $\lambda$ is of one of the forms as indicated.

(1) The formula follows from the fact that $\operatorname{dim} L^{\mathfrak{b}_{0}}(\lambda)=1$.

(2) By Corollary 4.3(2), we have

$$
\operatorname{ch} L^{\mathfrak{b}_{0}}(\lambda)=\operatorname{ch} I^{\mathfrak{b}_{0}}(\lambda)-\operatorname{ch} L^{\mathfrak{b}_{0}}(a \delta) .
$$

Then the formula follows from (1).

(3) By Corollary 4.3(2) and (3), we have the following complex:

$$
0 \longrightarrow L^{\mathfrak{b}_{0}}(a \delta) \longrightarrow I^{\mathfrak{b}_{0}}\left(\varepsilon_{1, a}\right) \longrightarrow \cdots \longrightarrow I^{\mathfrak{b}_{0}}\left(\varepsilon_{i-1, a}\right) \longrightarrow I^{\mathfrak{b}_{0}}\left(\varepsilon_{i, a}\right) \longrightarrow 0 .
$$

Then the character formula follows from (1) and (2).

(4) By Corollary 4.3(4), we have

$$
\operatorname{ch} L^{\mathfrak{b}_{0}}(\lambda)=\operatorname{ch} I^{\mathfrak{b}_{0}}(\lambda)-\operatorname{ch} L^{\mathfrak{b}_{0}}(\lambda-2 \delta)-\operatorname{ch} L^{\mathfrak{b}_{0}}(a \delta) .
$$

Then the desired formula holds.

(5) By Corollary 4.3.(5), we have

$$
\operatorname{ch} L^{\mathfrak{b}_{0}}\left(\varepsilon_{n, a}\right)=\operatorname{ch} I^{\mathfrak{b}_{0}}\left(\varepsilon_{n, a}\right)-\operatorname{ch} L^{\mathfrak{b}_{0}}\left(\varepsilon_{n-1, a}\right)-\operatorname{ch} L^{\mathfrak{b}_{0}}\left(\varepsilon_{n, 1, a-2}\right) .
$$

Then the character formula from (3) and (4). 
(6) By Corollary 4.3(5) and (6), we have the following complex:

$$
0 \longrightarrow L^{\mathfrak{b}_{0}}\left(\varepsilon_{n-1, b+2}\right) \longrightarrow I^{\mathfrak{b}_{0}}\left(\varepsilon_{n, 2, b}\right) \longrightarrow \cdots \longrightarrow I^{\mathfrak{b}_{0}}\left(\varepsilon_{n, a-1, b}\right) \longrightarrow I^{\mathfrak{b}_{0}}\left(\varepsilon_{n, a, b}\right) \longrightarrow 0 .
$$

Then the desired formula follows from (5).

(7) By Corollary 4.3(7), we have

$\operatorname{ch} L^{\mathfrak{b}_{0}}\left(\varepsilon_{n-1,1, a}\right)=\operatorname{ch} I^{\mathfrak{b}_{0}}\left(\varepsilon_{n-1,1, a}\right)-\operatorname{ch} L^{\mathfrak{b}_{0}}\left(\varepsilon_{n,-1, a}\right)-\operatorname{ch} L^{\mathfrak{b}_{0}}\left(\varepsilon_{n, 1, a-2}\right)-\operatorname{ch} L^{\mathfrak{b}_{0}}(a \delta)$.

Then the character formula follows from (4) and (6).

(8) By Corollary 4.3(7) and (8), we have the following complex:

$$
\begin{gathered}
0 \longrightarrow L^{\mathfrak{b}_{0}}\left(\varepsilon_{n,-1, a}\right) \longrightarrow I^{\mathfrak{b}_{0}}\left(\varepsilon_{n-1,1, a}\right) \longrightarrow \cdots \longrightarrow I^{\mathfrak{b}_{0}}\left(\varepsilon_{i+1,-(p-1), a}\right) \\
\longrightarrow \cdots \longrightarrow I^{\mathfrak{b}_{0}}\left(\varepsilon_{i+1,-1, a}\right) \longrightarrow I^{\mathfrak{b}_{0}}\left(\varepsilon_{i, 1, a}\right) \longrightarrow 0
\end{gathered}
$$

Then the character formula follows from (7).

(9) By Corollary 4.3(8) and (9), we have the following complex:

$$
0 \longrightarrow L^{\mathfrak{b}_{0}}\left(\varepsilon_{i+1,-1, b}\right) \longrightarrow I^{\mathfrak{b}_{0}}\left(\varepsilon_{i, 1, b}\right) \longrightarrow \cdots \longrightarrow I^{\mathfrak{b}_{0}}\left(\varepsilon_{i, a-1, b}\right) \longrightarrow I^{\mathfrak{b}_{0}}\left(\varepsilon_{i, a, b}\right) \longrightarrow 0 .
$$

Then the character formula follows from (8).

\section{Acknowledgments}

The authors are very grateful to the referee for his/her valuable suggestions, which help us to improve the original version.

\section{References}

[1] W. Bai and W. Liu, Superderivations for modular graded Lie superalgebras of Cartantype, Algebr. Represent. Theory 17 (2014), no. 1, 69-86.

[2] J. E. Humphreys, Representations of Semisimple Lie Algebras in the BGG Category $\mathscr{O}$, Graduate Studies in Mathematics 94, American Mathematical Society, Providence, RI, 2008.

[3] J. C. Jantzen, Representations of Lie algebras in prime characteristic, in: Representation Theories and Algebraic Geometry (Montreal, PQ, 1997), 185-235, NATO Adv. Sci. Inst. Ser. C Math. Phys. Sci. 514, Kluwer Acad., Dordrecht, 1998.

[4] V. G. Kac, Classification of infinite-dimensional simple linearly compact Lie superalgebras, Adv. Math. 139 (1998), no. 1, 1-55. 
[5] A. Lebedev, Analogs of the orthogonal, Hamiltonian, Possion, and contact Lie superalgebras in characteristic 2, J. Nonlinear Math. Phys. 17 (2010), suppl. 1, 217-251.

[6] D. Leites, J. Bernstein, S. Bouarroudj, B. Clarke, P. Grozman, A. Lebedev, D. Leites and I. Shchepochkina, Representation Theory, (Vol. 2. Nonholonomic Distributions in Quest for Simple Modular Lie Superalgebras), A. Salam School of Mathematical Sciences, Lahore, (2009)

[7] W.-Liu and Y. He, Finite-dimensional special odd Hamiltonian superalgebras in prime characteristic, Commun. Contemp. Math. 11 (2009), no. 4, 523-546.

[8] W. Liu and Y. Zhang, Finite-dimensional odd Hamiltonian superalgebras over a field of prime characteristic, J. Aust. Math. Soc. 79 (2005), no. 1, 113-130.

[9] V. Serganova, On representations of Cartan type Lie superalgebras, in: Lie Groups and Invariant Theory, 223-239, Amer. Math. Soc. Transl. Ser. 2 213, Adv. Math. Sci. 56, Amer. Math. Soc., Providence, RI, 2005.

[10] B. Shu and Y.-F. Yao, Character formulas for restricted simple modules of the special superalgebras, Math. Nachr. 285 (2012), no. 8-9, 1107-1116.

[11] B. Shu and C. Zhang, Restricted representations of the Witt superalgebras, J. Algebra 324 (2010), no. 4, 652-672.

[12] - Representations of the restricted Cartan type Lie superalgebra $W(m, n, 1)$, Algebr. Represent. Theory 14 (2011), no. 3, 463-481.

[13] Y.-F. Yao, On restricted representations of the extended special type Lie superalgebra $\bar{S}(m, n, 1)$, Monatsh. Math. 170 (2013), no. 2, 239-255.

[14] _ Non-restricted representations of simple Lie superalgebras of special type and Hamiltonian type, Sci. China Math. 56 (2013), no. 2, 239-252.

[15] Y.-F. Yao and B. Shu, Restricted representations of Lie superalgebras of Hamiltonian type, Algebr. Represent. Theory, 16 (2013), no. 3, 615-632.

[16] J. Yuan and W. Liu, Restricted Kac modules of Hamiltonian Lie superalgebras of odd type, Monatsh. Math. 178 (2015), no. 3, 473-488.

[17] Y. Zhang, Finite-dimensional Lie superalgebras of Cartan type over fields of prime characteristic, Chinese Sci. Bull. 42 (1997), no. 9, 720-724. 
Wende Liu

School of Mathematics and Statistics, Hainan Normal University, Haikou 571158, China E-mail address: wendeliu@ustc.edu.cn

Jixia Yuan and Shujuan Wang

School of Mathematical Sciences, Heilongjiang University, Harbin 150080, China

E-mail address: yuanjixia138@sina.com, wangshujuan619@163.com 